University of Wollongong

Research Online

Faculty of Engineering - Papers (Archive)

Faculty of Engineering and Information

Sciences

2007

\title{
Gate-tunable Ruderman-Kittel-Kasuya-Yosida interaction mediated by low- dimensional electrons with Rashba spin-orbit coupling
}

Pin Lyu

Jilin University

Ning-Ning Liu

Jilin University

Chao Zhang

University of Wollongong, czhang@uow.edu.au

Follow this and additional works at: https://ro.uow.edu.au/engpapers

Part of the Engineering Commons

https://ro.uow.edu.au/engpapers/3996

\section{Recommended Citation}

Lyu, Pin; Liu, Ning-Ning; and Zhang, Chao: Gate-tunable Ruderman-Kittel-Kasuya-Yosida interaction mediated by low-dimensional electrons with Rashba spin-orbit coupling 2007, 103910-1-103910-5. https://ro.uow.edu.au/engpapers/3996 


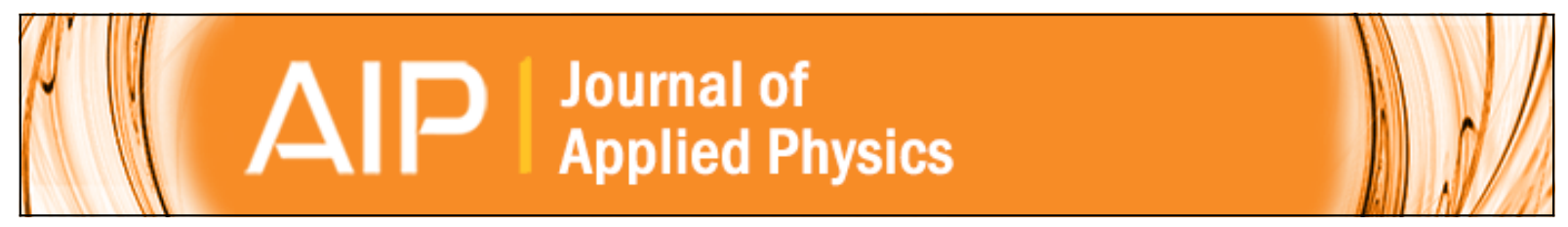

\section{Gate-tunable Ruderman-Kittel-Kasuya-Yosida interaction mediated by low-dimensional electrons with Rashba spin-orbit coupling}

Pin Lyu, Ning-Ning Liu, and Chao Zhang

Citation: Journal of Applied Physics 102, 103910 (2007); doi: 10.1063/1.2817405

View online: http://dx.doi.org/10.1063/1.2817405

View Table of Contents: http://scitation.aip.org/content/aip/journal/jap/102/10?ver=pdfcov

Published by the AIP Publishing

\section{Articles you may be interested in}

On the Ruderman-Kittel-Kasuya-Yosida interaction in graphene

AIP Conf. Proc. 1461, 24 (2012); 10.1063/1.4736868

Size effect of Ruderman-Kittel-Kasuya-Yosida interaction mediated by electrons in nanoribbons

J. Appl. Phys. 109, 083931 (2011); 10.1063/1.3575338

Twisted exchange interaction between localized spins in presence of Rashba spinorbit coupling

AIP Conf. Proc. 772, 1409 (2005); 10.1063/1.1994642

Ruderman-Kittel-Kasuya-Yosida interactions between spin distributions of arbitrary shape

J. Appl. Phys. 87, 5890 (2000); 10.1063/1.372557

Ruderman-Kittel-Kasuya-Yosida interaction between magnetic impurities in GaAs/AlAs quantum wells under strong electric field (abstract)

J. Appl. Phys. 81, 5308 (1997); 10.1063/1.364528

\section{A|P| Journal of}

Journal of Applied Physics is pleased to announce André Anders as its new Editor-in-Chief 


\title{
Gate-tunable Ruderman-Kittel-Kasuya-Yosida interaction mediated by low-dimensional electrons with Rashba spin-orbit coupling
}

\author{
Pin Lyu ${ }^{\text {a) }}$ and Ning-Ning Liu \\ Department of Physics, Jilin University, Changchun 130023, China \\ Chao Zhang \\ School of Engineering Physics, University of Wollongong, New South Wales 2522, Australia
}

(Received 31 July 2007; accepted 26 September 2007; published online 28 November 2007)

\begin{abstract}
The gate-controllable Ruderman-Kittel-Kasuya-Yosida (RKKY) magnetic interaction between the magnetic impurities embedded in one- and two-dimensional electron gas in the presence of Rashba spin-orbit coupling was analyzed theoretically. By using the eigenfunctions of the single-particle Rashba Hamiltonian as the basis of second quantization, we derived the RKKY interaction by fully taking into account the spin-orbit interaction. The dependence of the RKKY magnetic interaction on the Rashba spin-orbit coupling strength was obtained numerically. Our results clearly indicate that the RKKY interaction can be significantly modified by an applied voltage via the Rashba spin-orbit coupling. (C) 2007 American Institute of Physics. [DOI: 10.1063/1.2817405]
\end{abstract}

\section{INTRODUCTION}

The Ruderman-Kittel-Kasuya-Yosida (RKKY) magnetic interaction between nuclear spins and later between localized spins in metals mediated by conduction electrons was intensively studied in the $1950 \mathrm{~s}^{1-4}$ In recent years, there has been a renewed interest in the RKKY interaction as it plays an important role in giant magnetoresistance in multilayer structures $^{5}$ and ferromagnetism in diluted magnetic semiconductors. ${ }^{6}$ More recently, Craig et al. experimentally demonstrated the gate-controllable RKKY interaction between the localized spins in two quantum dots, each in contact with two-dimensional electron gas. ${ }^{7}$ The two quantized states of the spin of a single localized electron in a quantum dot can be considered as a quantum bit, and the extended nature of the controllable RKKY interaction between the coupled local spins has the potential application in building large-scale spin-based quantum computing and quantum computers. $^{8}$ It has stimulated the search for a different mechanism for the gate-controlled magnetic interaction both theoretically and experimentally.

The Rashba ${ }^{9}$ and Dresselhaus ${ }^{10}$ spin-orbit interactions in low-dimensional electron systems are currently attracting considerable interest due to their potential in controlling and manipulating the electronic spin and charge degrees of freedom in low-dimensional materials, a field known as spintronics. ${ }^{11}$ The Rashba spin-orbit interaction ${ }^{9}$ in twodimensional systems is caused by the structure inversion asymmetry originating from an electric field perpendicular to the plane of the systems. Experiments have confirmed that the strength of the Rashba spin-orbit interaction can be tuned by the gate voltage in InGaAs-based two-dimensional electron gas ${ }^{12,13}$ and GaAs-based two-dimensional hole gas. ${ }^{14}$ Through the gate-controllable Rashba spin-orbit interaction, the gate-tunable RKKY magnetic interaction between the magnetic impurities embedded in the Rashba spin-orbit

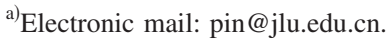

coupled electron systems is expected to be realized. By using Green's function method, Imamura et al. ${ }^{15}$ analytically obtained a twisted RKKY interaction between two localized spins of impurity in a two-dimensional electronic gas with Rashba spin-orbit coupling in the limit $q_{F} r \gg 1$, where $q_{F}$ is the Fermi wave vector and $r$ is the distance between two localized spins, in the twisted spin space where the spin quantization axis of one localized spin is rotated. It was pointed out that the twist angle can be controlled by gate voltage via the Rashba spin-orbit coupling according to their results. Without the above restriction of $q_{F} r \gg 1$, however, there is no clear indication available whether the strength of the Rashba spin-orbit coupling can significantly influence the variation of the magnitude and sign of the RKKY interaction. It is desirable to develop a different approach to treat the spin-orbit interaction in RKKY theory and further explore the effect of the Rashba spin-orbit coupling on the RKKY magnetic interaction.

In this paper we propose a transparent and direct treatment of the spin-orbit coupling, i.e., using the eigenfunctions of the single-particle Rashba Hamiltonian as the basis of second quantization, to calculate the RKKY interaction fully taking into account the spin-orbit interaction. The dependence of the RKKY magnetic interaction on the Rashba spinorbit coupling strength was obtained numerically. Our theoretical results clearly indicate that the RKKY interaction can be controlled by applied voltage via the Rashba spin-orbit coupling.

The paper is organized as follows. In Sec. II, the RKKY interaction mediated by one and two-dimensional spin-orbit coupled electrons is derived. In Sec. III, we present and discuss the numerical results of the gate-tunable RKKY interaction in the presence of the Rashba spin-orbit interaction. Finally, we conclude with a brief summary in Sec. IV.

\section{FORMALISM}

We consider two magnetic impurities with the localized spins $\mathbf{S}_{i}(i=1,2)$ embedded in the InGaAs-based two- 
dimensional electron gas, where there exists the Rashba spinorbit interaction. The localized spins interact with the conduction electrons via the $s$ - $d$ coupling. The Hamiltonian describing the above basic physics is written as

$$
\begin{aligned}
& H=H_{0}+H_{s-d}, \\
& H_{0}=\sum_{i=1}^{N}\left[\frac{\mathbf{p}_{i}^{2}}{2 m^{*}}+\frac{\lambda}{\hbar}\left(\sigma_{i x} p_{i y}-\sigma_{i y} p_{i x}\right)\right], \\
& H_{s-d}=-J \sum_{i=1,2} \sigma_{i} \cdot \mathbf{S}_{i},
\end{aligned}
$$

where the Rashba Hamiltonian $H_{0}$ describes the noninteracting two-dimensional conduction electrons in the presence of the Rashba spin-orbit interaction. ${ }^{9}$ The electrons are assumed to be confined in the $x-y$ plane. $H_{s-d}$ is the $s-d$ interaction between the conduction electrons and the localized $\operatorname{spin} \mathbf{S}_{i}$. $\lambda$ and $J$ are the Rashba spin-orbit interaction and the $s$ - $d$ interaction strengths, respectively. $\sigma_{i}$ is Pauli matrices and $m^{*}$ is the effective mass of the conduction electrons.

The eigenvalue and eigenfunction of the single-particle Rashba Hamiltonian are given by $E_{\mathbf{k} \mu}=\left(\hbar^{2} \mathbf{k}^{2} / 2 m^{*}\right)$ $+\mu \sqrt{w(\mathbf{k}) w^{*}(\mathbf{k})} \quad$ with $\quad w(\mathbf{k})=\lambda\left(i k_{x}+k_{y}\right), \quad$ and $\quad \Psi_{\mathbf{k} \mu}$ $=\psi_{\mathbf{k}}(x, y) \eta_{\mathbf{k} \mu}$ with $\psi_{\mathbf{k}}(x, y)=(1 / \sqrt{A}) e^{i\left(k_{x} x+k_{y} y\right)}$, and

$$
\eta_{\mathbf{k} \mu}=\frac{1}{\sqrt{2}}\left(\begin{array}{c}
1 \\
\frac{\mu w^{*}(\mathbf{k})}{\sqrt{w(\mathbf{k}) w^{*}(\mathbf{k})}}
\end{array}\right),
$$

where $A$ is the area of the two-dimensional system and $\mu$ is the energy level quantum number, $\mu= \pm 1$.

In order to fully take into account the spin-orbit interaction, we adopt a treatment where the eigenfunctions of the single-particle Rashba Hamiltonian are taken as the basis of the second quantization representation. This treatment of the spin-orbit coupling was also used to study the charge transport in the spin-orbit coupled electron systems. ${ }^{16,17}$ Now, in the second quantization presentation, we have the Hamiltonian $H=H_{0}+H_{s-d}$ as follows:

$$
H_{0}=\sum_{\mathbf{k} \mu} E_{\mathbf{k} \mu} c_{\mathbf{k} \mu}^{\dagger} c_{\mathbf{k} \mu}
$$

and

$$
H_{s-d}=-\frac{J}{A} \sum_{i=1,2, \mathbf{k}^{\prime} \mathbf{k} \mu \nu} e^{-i\left(\mathbf{k}^{\prime}-\mathbf{k}\right) \cdot \mathbf{r}_{i}} \eta_{\mathbf{k}^{\prime} \mu}^{+} \sigma_{i} \eta_{\mathbf{k} \nu} \cdot \mathbf{S}_{i} c_{\mathbf{k}^{\prime} \mu}^{\dagger} c_{\mathbf{k} \nu}
$$

where $c_{\mathbf{k} \mu}^{\dagger}$ and $c_{\mathbf{k} \mu}$ are the creation and annihilation operators of the conduction electron with wave vector $\mathbf{k}$ and level in$\operatorname{dex} \mu . \mathbf{r}_{i}$ is the position vector of the $i$ th localized spin. It is noted that the above formula can be generalized to include the Dresselhaus spin-orbit interaction. ${ }^{10}$ Here, we only focus on the effect due to the Rashba spin-orbit interaction.

Next, we derive the RKKY interaction by using the second-order perturbation theory. ${ }^{18}$ The effective Hamiltonian of RKKY interaction is given by

$$
H_{\mathrm{RKKY}}=\sum_{\Gamma} \frac{\left\langle\Gamma_{0}\left|H_{s-d}\right| \Gamma\right\rangle\left\langle\Gamma\left|H_{s-d}\right| \Gamma_{0}\right\rangle}{E_{\Gamma_{0}}-E_{\Gamma}},
$$

where $|\Gamma\rangle$ is the excited state with the energy $E_{\Gamma}$, and $\left|\Gamma_{0}\right\rangle$ is the ground state with the energy $E_{\Gamma_{0}}$. As usual, the excited state is taken as one particle-hole excited state $|\Gamma\rangle$ $=c_{\mathbf{q}^{\prime} \mu}^{\dagger} c_{\mathbf{q} \nu}\left|\Gamma_{0}\right\rangle$, where $\mathbf{q}^{\prime} \mu$ and $\mathbf{q} \nu$ satisfy $E_{\mathbf{q}^{\prime} \mu}>E_{F}$ and $E_{\mathbf{q} \nu}$ $<E_{F}$, respectively. From Eqs. (6) and (7), we obtain the effective Hamiltonian

$$
\begin{aligned}
H_{\mathrm{RKKY}}= & \frac{J^{2}}{A^{2}} \sum_{\mathbf{k}^{\prime} \mathbf{k} \mu \nu}\left[\eta_{\mathbf{k}^{\prime} \mu}^{+} \sigma_{1} \eta_{\mathbf{k} \nu} \cdot \mathbf{S}_{1} \eta_{\mathbf{k} \nu}^{+} \sigma_{2} \eta_{\mathbf{k}^{\prime} \mu} \cdot \mathbf{S}_{2}\right. \\
& \left.\times e^{i\left(\mathbf{k}-\mathbf{k}^{\prime}\right) \cdot \mathbf{r}}+\text { H.c. }\right] \frac{f\left(E_{\mathbf{k} \nu}\right)\left[1-f\left(E_{\mathbf{k}^{\prime} \mu}\right)\right]}{E_{\mathbf{k} \nu}-E_{\mathbf{k}^{\prime} \mu}},
\end{aligned}
$$

where $\mathbf{r}$ is the relative distance vector between the two localized spins given by $\mathbf{r}=\mathbf{r}_{1}-\mathbf{r}_{2}, f\left(E_{\mathbf{k} \nu}\right)$ is the Fermi-Dirac distribution, and H.c. stands for the Hermitian conjugate.

After inserting Eq. (4) into Eq. (8) and simply taking $\mathbf{r}$ along the $x$ axis without loss of generality, the resulting effective RKKY Hamiltonian is derived as follows:

$$
\begin{aligned}
H_{\mathrm{RKKY}}= & -\Phi(r) \mathbf{S}_{1} \cdot \mathbf{S}_{2}-\Phi_{1}(r)\left(S_{1}^{x} S_{2}^{x}-S_{1}^{y} S_{2}^{y}+S_{1}^{z} S_{2}^{z}\right) \\
& -\Phi_{2}(r)\left(\mathbf{S}_{1} \times \mathbf{S}_{2}\right)_{y},
\end{aligned}
$$

where the magnetic coupling strengths are given by

$$
\begin{aligned}
\Phi(r)= & -\frac{J^{2}}{A^{2}} \sum_{\mathbf{k}^{\prime} \mathbf{k} \mu \nu} \frac{\operatorname{Re}\left[e^{i\left(\mathbf{k}-\mathbf{k}^{\prime}\right) \cdot \mathbf{r}}\right]}{E_{\mathbf{k} \nu}-E_{\mathbf{k}^{\prime} \mu}} f\left(E_{\mathbf{k} \nu}\right)\left[1-f\left(E_{\mathbf{k}^{\prime} \mu}\right)\right], \\
\Phi_{1}(r)= & \frac{J^{2}}{A^{2}} \sum_{\mathbf{k}^{\prime} \mathbf{k} \mu \nu} \frac{\operatorname{Re}\left[e^{i\left(\mathbf{k}-\mathbf{k}^{\prime}\right) \cdot \mathbf{r}}\right]}{E_{\mathbf{k} \nu}-E_{\mathbf{k}^{\prime} \mu}} \mu \nu \\
& \times \frac{k_{x} k_{x}^{\prime}}{k k^{\prime}} f\left(E_{\mathbf{k} \nu}\right)\left[1-f\left(E_{\mathbf{k}^{\prime} \mu}\right)\right], \\
\Phi_{2}(r)= & -\frac{J^{2}}{A^{2}} \sum_{\mathbf{k}^{\prime} \mathbf{k} \mu \nu} \frac{\operatorname{Im}\left[e^{i\left(\mathbf{k}-\mathbf{k}^{\prime}\right) \cdot \mathbf{r}}\right]}{E_{\mathbf{k} \nu}-E_{\mathbf{k}^{\prime} \mu}} \\
& \times\left(\mu_{x} \frac{k_{x}^{\prime}}{k^{\prime}}-\nu \frac{k_{x}}{k}\right) f\left(E_{\mathbf{k} \nu}\right)\left[1-f\left(E_{\mathbf{k}^{\prime} \mu}\right)\right] .
\end{aligned}
$$

Here, $\mathbf{k}=\left(k_{x}, k_{y}\right)=(k \cos \theta, k \sin \theta), \mathbf{k}^{\prime}=\left(k_{x}^{\prime}, k_{y}^{\prime}\right)=\left(k^{\prime} \cos \theta^{\prime}\right.$, $\left.k^{\prime} \sin \theta^{\prime}\right)$, and $\left(\mathbf{k}-\mathbf{k}^{\prime}\right) \cdot \mathbf{r}=k r \cos \theta-k^{\prime} r \cos \theta^{\prime}$. The above formulas are rigorous and can be applied to systems of arbitrary density and at any temperature. Equation (11) does not involve $k_{y} k_{y}^{\prime}$ in the factor $k_{x} k_{x}^{\prime} / k k^{\prime}$. This is because the electronic motion perpendicular to the relative distance vector between the two localized spins has no contribution to the RKKY interaction, while the RKKY interaction originates from the electronic motion along the relative distance vector.

It should be mentioned here that Eqs. (9)-(12) are derived by setting the vector $\mathbf{r}$ along the $x$ axis. If we take the vector $\mathbf{r}$ to be along an arbitrary direction, it is necessary to transform the localized spins $\mathbf{S}_{i}$ and the wave vector $\mathbf{k}$ from the original coordinate system to another coordinate system whose $x$ axis is along the vector $\mathbf{r}$. Then, we arrive at the same result as that given by the above Eqs. (9)-(12), which 
are independent of the direction of the vector $\mathbf{r}$. This also gives the explanation that the RKKY interaction coupling strengths Eqs. (10)-(12) are only dependent on the distance of the two localized spins when there is Rashba spin-orbit coupling.

From our results, we can give the twisted exchange interaction obtained in Ref. 15. By using the transformation $\mathbf{k}+\mu\left(k_{R} / k\right) \mathbf{k}=\mathbf{q}$, where $k_{R}=m^{*} \lambda / \hbar^{2}$, one can get the same exact twisted RKKY interaction in one dimension from Eqs. (9)-(12) as that of Ref. 15. Under the approximations $k_{x} / k$ $\approx 1, k_{x}^{\prime} / k^{\prime} \approx 1$, and in the limit $2 q_{F} r \gg 1$, the twisted RKKY interaction in two dimensions in Ref. 15 is also obtained from our above formulas. Our treatment of the spin-orbit interaction in RKKY theory allows us to give the general formulation of the RKKY interaction in the presence of the spin-orbit coupling.

To get more physical insight, we rewrite the RKKY Hamiltonian Eq. (9) as

$$
\begin{aligned}
H_{\mathrm{RKKY}}= & -\Phi_{x z}(r)\left(S_{1}^{x} S_{2}^{x}+S_{1}^{z} S_{2}^{z}\right)+\Phi_{y}(r) S_{1}^{y} S_{2}^{y} \\
& -\Phi_{2}(r)\left(\mathbf{S}_{1} \times \mathbf{S}_{2}\right)_{y},
\end{aligned}
$$

with $\Phi_{x z}(r)=\Phi(r)+\Phi_{1}(r)$ and $\Phi_{y}(r)=\Phi_{1}(r)-\Phi(r)$. It is very clear that the rewritten RKKY Hamiltonian consists of twodimensional Heisenberg interaction in the $x-z$ plane, Ising interaction along the $y$ direction, and the $y$ component of the well-known Dzyaloshinsky-Moriya ${ }^{19,20}$ interaction. The Dzyaloshinsky-Moriya interaction disappears if the two localized spins are paralleled or antiparalleled.

In the absence of the Rashba spin-orbit coupling, i.e., $\lambda=0$, Eq. (10) is reduced to $\Phi^{\mathrm{cl}}(r)=-4 J^{2} / A^{2} \Sigma_{\mathbf{k}^{\prime} \mathbf{k}}(\{\cos [(\mathbf{k}$ $\left.\left.\left.\left.-\mathbf{k}^{\prime}\right) \cdot \mathbf{r}\right]\right\} /\left(E_{\mathbf{k}}-E_{\mathbf{k}^{\prime}}\right)\right) f\left(E_{\mathbf{k}}\right)\left[1-f\left(E_{\mathbf{k}^{\prime}}\right)\right]$, and the other coupling strengths Eqs. (11) and (12) disappear. It follows that the Hamiltonian Eq. (9) is reduced to the conventional RKKY interaction with spin degeneracy, $H_{\mathrm{RKKY}}^{\mathrm{cl}}=-\Phi^{\mathrm{cl}}(r) S_{1} \cdot \mathbf{S}_{2}$. The first term in the RKKY Hamiltonian Eq. (9) is the direct extension of the conventional RKKY interaction, and the presence of the other terms is due to the spin-orbit coupling. The sums in Eqs. (10)-(12) involve two Fermi wave vectors, which directly depend on the Rashba spin-orbit coupling strength given by $k_{F}^{ \pm}=\mp\left(\lambda m^{*} / \hbar^{2}\right)$ $+\sqrt{\left(2 m^{*} / \hbar^{2}\right) E_{F}+\left(\lambda^{2} m^{*} / \hbar^{4}\right)}$, where $E_{F}$ is Fermi energy. As a result, the present RKKY magnetic interactions Eqs. (9)-(12) can be adjusted by the Rashba spin-orbit coupling strength, and further by the applied voltage across the twodimensional electron system.

For the one-dimensional situation, the spinor part of the wave functions of the single-particle Rashba Hamiltonian is independent of the conduction electron momentum. From Eqs. (9)-(12), the one-dimensional effective RKKY Hamiltonian is given by $H_{\mathrm{RKKY}}^{1 \mathrm{D}}=-\Phi^{1 \mathrm{D}}(r) \mathbf{S}_{1} \cdot \mathbf{S}_{2}-\Phi_{1}^{1 \mathrm{D}}(r)\left(S_{1}^{x} S_{2}^{x}\right.$ $\left.-S_{1}^{y} S_{2}^{y}+S_{1}^{z} S_{2}^{z}\right)-\Phi_{2}^{1 \mathrm{D}}(r)\left(\mathbf{S}_{1} \times \mathbf{S}_{2}\right)_{y}$ where the $\Phi^{1 \mathrm{D}}(r), \Phi_{1}^{1 \mathrm{D}}(r)$, and $\Phi_{2}^{1 \mathrm{D}}(r)$ are the corresponding results of Eqs. (10)-(12) with the one-dimensional length $L$ instead of the area $A, k_{y}$ $=0, k_{x}=k$, and $k_{x}^{\prime}=k^{\prime}$.

\section{RESULT AND DISCUSSION}

Now, we present our numerical results of the dependence of the RKKY interaction on the Rashba spin-orbit coupling
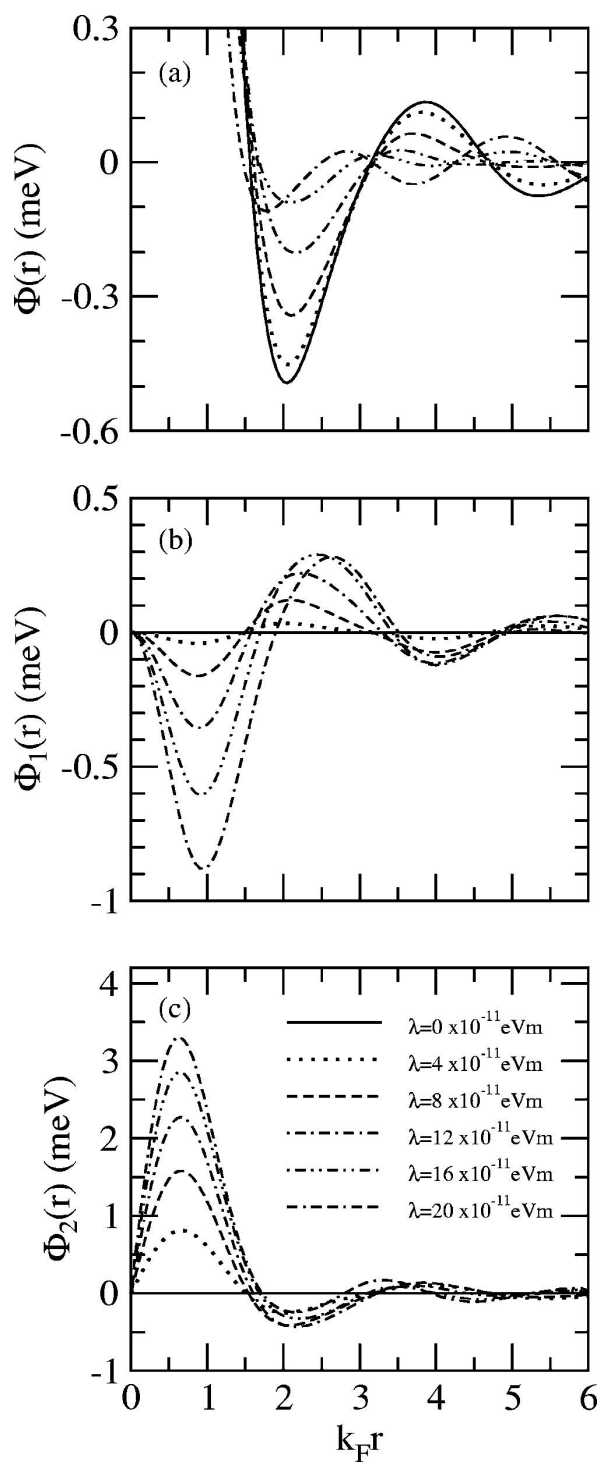

FIG. 1. Indirect RKKY magnetic interaction energies vs the distance between two localized spins at several different Rashba spin-orbit coupling strengths in two dimensions.

strength in two dimensions at zero temperature. We chose the following typical material parameters: $m^{\star}=0.052 m_{e}, n=1.0$ $\times 10^{12} \mathrm{~cm}^{-2}$ appropriate for two-dimensional electron gas in the inverted $\operatorname{In}_{x} \mathrm{Ga}_{1-x} \mathrm{As} / \mathrm{In}_{y} \mathrm{Al}_{1-y}$ As heterostructure with the Rashba spin-orbit coupling. ${ }^{12}$ Also, we used $J=1.0 \mathrm{eV} \mathrm{nm}^{2}$ for the $s-d$ coupling strength. In the figure we take $k_{F} r$ as the dimensionless distance between the two localized spins, where $k_{F}$ is the Fermi wave vector without the spin-orbit interaction. The RKKY interaction energies $\Phi(r), \Phi_{1}(r)$, and $\Phi_{2}(r)$ vs $k_{F} r$ at different Rashba spin-orbit coupling strengths are plotted in Fig. 1.

For a system without any spin-orbit coupling, the numerical results show that $\Phi(r)$ is deduced to the conventional RKKY interaction energy and $\Phi_{1}(r)$ and $\Phi_{2}(r)$ disappear, which are in agreement with the analytical results given in Eqs. (10)-(12). In the small Rashba spin-orbit coupling strength region, the energy coupling strength $\Phi(r)$ oscillates and decays with the relative distance of two coupled local spins like the behavior of the conventional RKKY interac- 


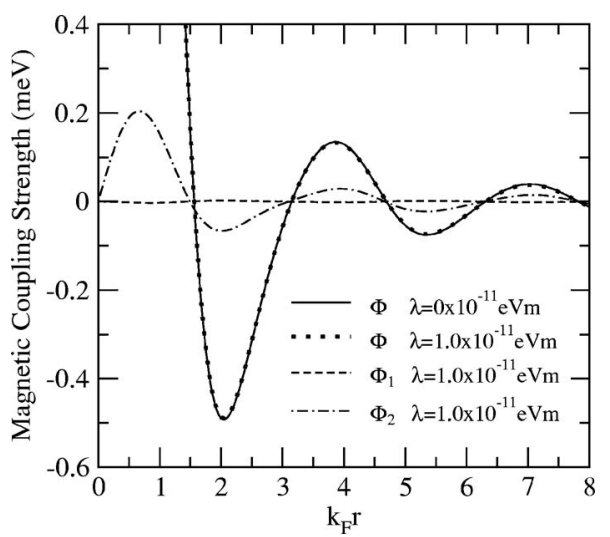

FIG. 2. Indirect RKKY magnetic interaction energies vs the distance between two localized spins at the Rashba spin-orbit coupling strength obtained in Ref. 12 in two dimensions.

tion, and its amplitude decreases with the Rashba spin-orbit interaction strength. However, for large values of the spinorbit coupling strength, for example, $\lambda=20 \times 10^{11} \mathrm{eV} \mathrm{m}$, the $\Phi(r)$ loses the decaying character and becomes nearly oscillatory with the distance in the regime of $2<k_{F} r<6$. This is a quite different behavior as compared to that of the conventional RKKY interaction. We explain this anomalous behavior as follows: The conventional RKKY interaction is an oscillating and decaying function of $2 k_{F} r$ and only involves one Fermi wave vector due to spin degeneracy. Its spatial dependence is proportionable to $\sin \left(2 k_{F} r\right) /\left(2 k_{F} r\right)^{2}$ in two dimensions when $2 k_{F} r \gg 1 .^{4,21}$ In the presence of the spin-orbit coupling, the band of conduction electrons splits and at some $k$ value, the spin degeneracy is left. There are two Fermi wave vectors $k_{F}^{+}$and $k_{F}^{-}$associated with the sum of $\Phi(r)$, and $\Phi(r)$ has four different terms. Each of them is a decaying function with its own oscillating behavior. Thus, the contributions of the different oscillation functions to $\Phi(r)$ result in the enhancement or the suppression of amplitude at different values of the distance between the two localized spins for the strong spin-orbit coupling strengths. In Fig. $1, \Phi_{1}(r)$ and $\Phi_{2}(r)$ become stronger with increasing the spin-orbit coupling strength in the regimes of $k_{F} r<3.5$ and $k_{F} r<1.5$, respectively. The similar enhanced and suppressed behavior also occurs in $\Phi_{1}(r)$ at $k_{F} r>3.5$ and in $\Phi_{2}(r)$ at $k_{F} r>1.5$, where their amplitude is small. Though there is the enhancement of amplitude, the long-distance behavior of $\Phi(r)$, $\Phi_{1}(r)$, and $\Phi_{2}(r)$ has the tendency of decay as that of the usual RKKY interaction. Another important feature is that the magnitudes of $\Phi_{1}(r)$ and $\Phi_{2}(r)$ has their maximum at $k_{F} r \sim 1$, where the effect of the spin-orbit coupling reaches saturation.

In Fig. 2 we plotted the RKKY magnetic coupling strengths $\Phi(r), \Phi_{1}(r)$, and $\Phi_{2}(r)$ vs $k_{F} r$ at the Rashba spinorbit coupling strength $\lambda=1.0 \times 10^{-11} \mathrm{eV}$ m experimentally obtained in Ref. 12. In this situation, the results show that $\Phi_{2}(r)$ does emerge; however, $\Phi(r)$ has almost no change compared with the conventional RKKY interaction and $\Phi_{1}(r)$ does not appear obviously. The observed maximum values of the Rashba spin-orbit coupling are about 4 $\times 10^{-11} \mathrm{eV} \mathrm{m}$ as reported in Ref. 22, which was used in Fig. 1. Since the Rashba spin-orbit coupling strength available now does not saturate in the applied gate voltage range in the present experiment, it will be possible to obtain a larger Rashba spin-orbit coupling strength. ${ }^{23}$ Therefore, our theoretical results shown in Figs. 1 and 2 can be useful.

The variation of the amplitude and the sign of $\Phi(r)$, $\Phi_{1}(r)$, and $\Phi_{2}(r)$ with gate-tunable Rashba spin-orbit coupling strength clearly indicates that the RKKY interaction can be significantly controlled by applied voltage via the Rashba spin-orbit coupling. This gate-controllable RKKY interaction has a different mechanism from that of two spins localized in two quantum dots, as reported in Ref. 7. The latter is realized by changing the gate-controlled electron number in the quantum dot and the gate-controlled coupling of the quantum dot to the two-dimensional electron gas.

In addition, the RKKY interaction mediated by onedimensional electrons with Rashba spin-orbit coupling was numerically calculated. The amplitude and the sign of $\Phi^{1 \mathrm{D}}(r), \Phi_{1}^{1 \mathrm{D}}(r)$, and $\Phi_{2}^{1 \mathrm{D}}(r)$ are still sensitive to the Rashba spin-orbit coupling strength, and the enhancement and suppression of amplitude become acute compared with the twodimensional situation.

Finally, we pay attention to the magnetic structure. In comparison to the usual RKKY interaction, i.e., Heisenberg coupling, the present RKKY interaction has Heisenberg, Ising, and Dzyaloshinsky-Moriya coupling. This can lead to rich magnetic structures due to the presence of the Rashba spin-orbit coupling.

\section{SUMMARY}

We presented a different approach to derive the RKKY interaction in the presence of the spin-orbit coupling by taking the eigenfunctions of the single-particle Rashba Hamiltonian as the basis of second quantization representation. Our theoretical results demonstrate that the RKKY magnetic interactions can be significantly controlled by the applied voltage via the Rashba spin-orbit coupling. The experimental observation of the above effect can be performed in the magnetic impurities embedded in the InGaAs-based twodimensional electron gas or the GaAs-based twodimensional hole gas with Rashba spin-orbit coupling.

\section{ACKNOWLEDGMENTS}

This work was supported by the National Science Foundation of China (Grant Nos. 10304006 and J0730311) and the Australian Research Council.

\footnotetext{
${ }^{1}$ M. A. Ruderman and C. Kittel, Phys. Rev. 96, 99 (1954).

${ }^{2}$ T. Kasuya, Prog. Theor. Phys. 16, 45 (1956).

${ }^{3}$ K. Yosida, Phys. Rev. 106, 893 (1957).

${ }^{4}$ C. Kittel, in Solid State Physics, edited by F. Seitz, D. Turnbull, and H. Ehrenreich (Academic, New York, 1968), Vol. 22, p. 1.

${ }^{5}$ S. S. P. Parkin, N. More, and K. P. Roche, Phys. Rev. Lett. 64, 2304 (1990).

${ }^{6}$ T. Dietl, H. Ohno, J. Cibert, and D. Ferrand, Science 287, 1019 (2000).

${ }^{7}$ N. J. Craig, J. M. Taylor, E. A. Lester, C. M. Marcus, M. P. Hanson, and A. C. Gossard, Science 304, 565 (2004).

${ }^{8}$ L. I. Glazman and R. C. Ashoori, Science 304, 524 (2004).

${ }^{9}$ E. I. Rashba, Sov. Phys. Solid State 2, 1109 (1960); Y. A. Bychkov and E. I. Rashba, J. Phys. C 17, 6039 (1984).

${ }^{10}$ G. Dresselhaus, Phys. Rev. 100, 580 (1955).

${ }^{11}$ S. A. Wolf, D. D. Awschalom, R. A. Buhrman, J. M. Daughton, S. von
} 
Molnar, M. L. Roukes, A. Y. Chtchelkanova, and D. M. Treger, Science 294, 1488 (2001)

${ }^{12}$ J. Nitta, T. Akazaki, H. Takayanagi, and T. Enoki, Phys. Rev. Lett. 78, 1335 (1997).

${ }^{13}$ G. Engels, J. Lange, Th. Schäpers, and H. Lüth, Phys. Rev. B 55, R1958 (1997).

${ }^{14}$ J. P. Lu, J. B. Yau, S. P. Shukla, M. Shayegan, L. Wissinger, U. Rössler, and R. Winkler, Phys. Rev. Lett. 81, 1282 (1998).

${ }^{15}$ H. Imamura, P. Bruno, and Y. Utsumi, Phys. Rev. B 69, 121303 (2004).
${ }^{16}$ C. Zhang and Z. S. Ma, Phys. Rev. B 71, 121307(R) (2005).

${ }^{17}$ P. Lyu and C. Zhang, Nanotechnology 18, 475403 (2007).

${ }^{18}$ C. H. Ziener, S. Glutsch, and F. Bechstedt, Phys. Rev. B 70, 075205 (2004).

${ }^{19}$ I. Dzyaloshinsky, J. Phys. Chem. Solids 4, 241 (1958).

${ }^{20}$ T. Moriya, Phys. Rev. Lett. 4, 228 (1960).

${ }^{21}$ M. T. Béal-Monod, Phys. Rev. B 36, 8835 (1987).

${ }^{22}$ D. Grundler, Phys. Rev. Lett. 84, 6074 (2000).

${ }^{23}$ J. Nitta, NTT Tech. Rev. 2, 31 (2004). 